\title{
Thermodynamic Analysis of the Static Spherically Symmetric Field Equations in Rastall Theory
}

\author{
Hooman Moradpour ${ }^{1}$ and Ines G. Salako ${ }^{2,3,4}$ \\ ${ }^{1}$ Research Institute for Astronomy and Astrophysics of Maragha (RIAAM), P.O. Box 55134-441, Maragha, Iran \\ ${ }^{2}$ African Institute for Mathematical Sciences (AIMS), 6 Melrose Road, Muizenberg 7945, South Africa \\ ${ }^{3}$ Institut de Mathématiques et de Sciences Physiques (IMSP), Université de Porto-Novo, 01 BP 613 Porto-Novo, Benin \\ ${ }^{4}$ Département de Physique, Université d’Agriculture de Kétou, BP 13 Kétou, Benin
}

Correspondence should be addressed to Hooman Moradpour; hn.moradpour@gmail.com

Received 20 July 2016; Accepted 19 September 2016

Academic Editor: Elias C. Vagenas

Copyright (C) 2016 H. Moradpour and I. G. Salako. This is an open access article distributed under the Creative Commons Attribution License, which permits unrestricted use, distribution, and reproduction in any medium, provided the original work is properly cited. The publication of this article was funded by SCOAP ${ }^{3}$.

\begin{abstract}
The restrictions on the Rastall theory due to application of the Newtonian limit to the theory are derived. In addition, we use the zero-zero component of the Rastall field equations as well as the unified first law of thermodynamics to find the Misner-Sharp mass content confined to the event horizon of the spherically symmetric static spacetimes in the Rastall framework. The obtained relation is calculated for the Schwarzschild and de-Sitter back holes as two examples. Bearing the obtained relation for the MisnerSharp mass in mind together with recasting the one-one component of the Rastall field equations into the form of the first law of thermodynamics, we obtain expressions for the horizon entropy and the work term. Finally, we also compare the thermodynamic quantities of system, including energy, entropy, and work, with their counterparts in the Einstein framework to have a better view about the role of the Rastall hypothesis on the thermodynamics of system.
\end{abstract}

\section{Introduction}

For the first time, Jacobson could use thermodynamics to derive the Einstein field equations [1]. The Bekenstein entropy is the backbone of Jackobson's approach confirming that the Einstein theory corresponds with the Bekenstein entropy [1]. Generalization of his approach to $f(R)$ gravity shows that the terms other than the Einstein tensor in the gravitational field equations produce entropy and therefore modify the Bekenstein limit of the horizon entropy [2]. In fact, such terms and thus their corresponding entropy production terms are the signals of the nonequilibrium thermodynamic aspects of spacetime [2].

In order to study the mutual relation between gravity and thermodynamics, we need a proper energy definition, and it seems that the generalization of the Misner-Sharp mass in various theories is a suitable candidate for this aim [3-10]. It is also shown that, in various gravitational theory, if the gravitational field equations are considered as the first law of thermodynamics, then we can find an expression for the horizon entropy in the spherically symmetric static spacetimes [710]. This approach is used to investigate the mutual relation between the gravitational field equations and the system thermodynamic properties, such as entropy, in various gravitational theories [11-23]. In all of the above mentioned attempts [1-23], authors have only studied theories in which geometry and matter fields are coupled to each other in a minimal way, and therefore, the energy-momentum conservation law is met in their studies. In fact, in their studies, the lagrangian system is equal to total sum of its lagrangian constituents including the geometry and the matter fields.

Rastall [24] and curvature-matter coupling [25-29] theories are two gravitational theories in which geometry and matter fields are coupled to each other in a nonminimal way. In these theories, the lagrangian of a gravitational system is not just a simple sum of the geometry and the matter fields lagrangians $[29,30]$. For these theories, the energy-momentum conservation law is not always valid, and in fact, the divergence of the energy-momentum tensor is proportional 
to the derivative of Ricciscalar. This mutual relation between the divergence of the energy-momentum tensor and Ricciscalar is the backbone of the Rastall theory allowing a flux of energy between the energy-momentum source and the geometry. The Rastall hypothesis also modifies the Einstein field equations, a result that increases the hopes to describe the current phase of the universe expansion [31]. From a classical point of view, Rastall's theory may be supported by the matter production process in the cosmos [32-35]. The mutual relation between the Rastall cosmology and thermodynamics has recently been studied [36]. More studies on this theory can also be found in [37-51].

Here, we are interested in studying the mutual relation between the thermodynamics first law and the Rastall field equations. In fact, we want to study the effects of the Rastall hypothesis on the thermodynamic properties of the spherically symmetric static spacetimes. In order to achieve this goal, generalizing the Misner-Sharp mass of the spherically symmetric static spacetimes to Rastall theory, we find an expression for the horizon entropy of the spherically symmetric static spacetimes. We also compare the thermodynamic quantities of system, including energy, entropy, and work, with their counterparts in the Einstein framework to have a better view about the role of the Rastall hypothesis on the thermodynamics of system. The $G=\hbar=c=1$ units are considered in this paper.

The paper is organized as follows. In the next section, after referring to the Rastall theory and the Newtonian limit constraints on this theory parameters, we use the unified first law of thermodynamics together with the zero-zero component of the Rastall field equations to find the MisnerSharp mass confined to event horizon of the static spherically symmetric spacetimes in Rastall theory. Thereinafter, in the third section, recasting the one-one component of the Rastall field equations into the form of the first law of thermodynamics, we find an expression for the horizon entropy. The comparison of the obtained thermodynamic quantities with their counterparts in the Einstein general relativity is also addressed in this section. The last section is devoted to concluding remarks.

\section{Rastall Field Equations and the Misner-Sharp Mass}

For Rastall's original field equations, we have [24]

$$
T_{; \mu}^{\mu \nu}=\lambda R^{, v}
$$

which finally leads to

$$
G_{\mu \nu}+\kappa \lambda g_{\mu \nu} R=\kappa T_{\mu \nu}
$$

where $\lambda$ and $\kappa$ are Rastall's parameter and Rastall's gravitational coupling constant, respectively. As Rastall has been shown [24], this equation leads to $R(4 \kappa \lambda-1)=T$, indicating that, since $T$ is not always zero, the $\kappa \lambda=1 / 4$ case is not allowed in this theory. Besides, it is also shown that if we use the Newtonian limit and define the Rastall dimensionless parameter $\gamma=\kappa \lambda$, then for the Rastall gravitational coupling constant $(\kappa)$ and the Rastall original parameter $(\lambda)$ we have

$$
\begin{aligned}
& \kappa=\frac{4 \gamma-1}{6 \gamma-1} 8 \pi, \\
& \lambda=\frac{\gamma(6 \gamma-1)}{(4 \gamma-1) 8 \pi},
\end{aligned}
$$

indicating that the Einstein result $(\kappa=8 \pi)$ is obtainable in the appropriate limit $\lambda=0$ which is parallel to the $\gamma=0$ limit [51]. It is useful to mention here that as this equation shows, since the Rastall gravitational coupling constant diverges at $\gamma=1 / 6$, the $\gamma=1 / 6$ case is not also allowed. Finally, Rastall's field equations can be written as [51]

$$
G_{\mu}^{\nu}+\gamma g_{\mu}^{\nu} R=\frac{4 \gamma-1}{6 \gamma-1} 8 \pi T_{\mu}^{\nu},
$$

which leads to $R(6 \gamma-1)=8 \pi T$ meaning that, in agreement with (3), the $\gamma=\kappa \lambda=1 / 6$ case is not allowed in this theory. Moreover, as it is obvious from (3), $\lambda$ diverges at $\gamma=1 / 4$, and thus, the $\gamma=1 / 4$ case is not also allowed. Therefore, Newtonian limit indicates that, in fact, both the $\gamma=1 / 6$ and $\gamma=1 / 4$ cases are not allowed. In order to study the mutual relation between the first law of thermodynamics and the Rastall field equations, we need to generalize the MisnerSharp energy in this theory $[3,5,6]$. It is worthwhile mentioning that one can use either the conserved charge method or the unified first law of thermodynamics in order to find the Misner-Sharp mass in a gravitational theory $[5,6]$. Here, since we are interested in having a fully thermodynamic analysis, we use the unified first law of thermodynamics to obtain an expression for the Misner-Sharp mass. Consider a spherically symmetric static spacetime, with a horizon located at $r_{h}$, described by

$$
d s^{2}=-f(r) d t^{2}+\frac{d r^{2}}{f(r)}+r^{2} d \Omega^{2}
$$

and filled by a source of energy-momentum $T_{\gamma}^{\mu}$, the work density and energy supply vector are defined as

$$
\begin{aligned}
& W=-\frac{h^{a b} T_{a b}}{2}, \\
& \Psi_{a}=T_{a}^{b} \partial_{b} r+W \partial_{a} r,
\end{aligned}
$$

respectively. In the above equations, $d \Omega^{2}$ is the line element on the two-dimensional sphere with radius $r$, and $h_{a b}$ is metric on the two-dimensional hypersurface of $(t, r)$. In order to generalize the Misner-Sharp mass definition to the Rastall theory, we follow the approach of [6] and assume that the unified first law (UFL) of thermodynamics is valid meaning that

$$
d E \equiv A \Psi_{a} d x^{a}+W d V
$$

in which $x^{a}$ denotes the coordinate on a two-dimensional hypersurface of metric $h_{a b}$ and $A$ is the area of the system 
boundary and therefore $A=4 \pi r^{2}$. It is straightforward to show that

$$
d E=\rho\left(4 \pi r^{2}\right) d r .
$$

Using the zero-zero component of (4) in rewriting this equation, we obtain

$$
\begin{aligned}
d E & =\frac{6 \gamma-1}{2(4 \gamma-1)}\left[(1-2 \gamma)\left(1-\frac{d(r f(r))}{d r}\right)\right. \\
& \left.+\gamma \frac{d\left(r^{2} f^{\prime}(r)\right)}{d r}\right] d r,
\end{aligned}
$$

which finally leads to

$$
E=\frac{(6 \gamma-1) r}{2(4 \gamma-1)}\left[(1-2 \gamma)(1-f(r))+\gamma r f^{\prime}(r)\right],
$$

where I denotes derivative with respect to $r$, for the energy confined to radius $r$. In obtaining this result, we used $r f^{\prime}(r)=$ $d(r f(r)) / d r-f(r)$. For a black hole with an event horizon located at $r_{h}$, since $f\left(r_{h}\right)=0$, we find

$$
E=\frac{6 \gamma-1}{2(4 \gamma-1)}\left[(1-2 \gamma) r_{h}+\gamma r_{h}^{2} f^{\prime}\left(r_{h}\right)\right] .
$$

In fact, it is the Misner-Sharp mass content confined to the mentioned horizon in the Rastall framework. The above equation can also be written as $E=E_{0}(1+\Gamma)$, where

$$
\begin{aligned}
E_{0} & =\frac{r_{h}}{2}, \\
\Gamma & =\frac{\gamma}{4 \gamma-1}\left[(6 \gamma-1) r_{h} f^{\prime}\left(r_{h}\right)+4(1-3 \gamma)\right] .
\end{aligned}
$$

It is also worthwhile mentioning that, in the $\gamma \rightarrow 0$ limit, we have $\widetilde{E} \equiv E_{0} \Gamma \rightarrow 0$ leading to $E \rightarrow E_{0}$ which is nothing but the Misner-Sharp mass content of the Einstein theory $[3,7]$, meaning that, as expected, the Einstein result is recovered in the appropriate limit of $\gamma \rightarrow 0$. The $E_{0} \Gamma$ term comes from the Rastall original hypothesis (1) that admits a mutual energy exchange between spacetime and energy-momentum source supporting the geometry. This hypothesis couples the geometry and matter fields in a nonminimal way and leads to a covariant nonconservation of Rastall gravity. As two examples, calculations for the value of $\Gamma$ in the Schwarzschild and de-Sitter spacetimes lead to

$$
\begin{aligned}
f(r) & =1-\frac{2 m}{r} \longrightarrow \\
r_{h} & =2 m \Longrightarrow \\
\Gamma_{\text {sch }} & =\frac{3 \gamma(1-2 \gamma)}{4 \gamma-1}, \\
f(r) & =1-\Lambda r^{2} \longrightarrow \\
r_{h} & =\frac{1}{\sqrt{\Lambda}} \Longrightarrow \\
\Gamma_{\mathrm{deS}} & =-6 \gamma,
\end{aligned}
$$

respectively. Finally, it is worthwhile mentioning that, in order to have positive energy, we should have $\Gamma \geq-1$ leading to the $r_{h} f^{\prime}\left(r_{h}\right) \leq(1+4 \gamma(3 \gamma-2)) / \gamma(6 \gamma-1)$ condition for the Rastall dimensionless parameter.

\section{Horizon Entropy}

Here, following the approach of [7], we recast the one-one component of the Rastall field equations to the form of the first law of thermodynamics and use the result of previous section to get the horizon entropy. The one-one component of (4) yields

$$
G_{1}^{1}+\gamma R=\frac{4 \gamma-1}{6 \gamma-1} 8 \pi T_{1}^{1},
$$

where $T_{1}^{1} \equiv P(r)$ is the radial pressure of the energymomentum source [7], and therefore, it finally takes the

$$
\begin{gathered}
P(r)=\frac{6 \gamma-1}{(4 \gamma-1) 8 \pi}\left(\frac{1}{r^{2}}\left[r f^{\prime}(r)-1+f(r)\right]\right. \\
\left.-\frac{\gamma}{r^{2}}\left[r^{2} f^{\prime \prime}(r)+4 r f^{\prime}(r)-2+2 f(r)\right]\right)
\end{gathered}
$$

form. Here, prime ( $I$ ) denotes the derivative with respect to radius $(r)$. On the event horizon, $f\left(r_{h}\right)=0$ and therefore,

$$
\begin{gathered}
P\left(r_{h}\right)=\frac{6 \gamma-1}{(4 \gamma-1) 8 \pi}\left(\frac{1}{r_{h}^{2}}\left[r_{h} f^{\prime}\left(r_{h}\right)-1\right]\right. \\
\left.-\frac{\gamma}{r_{h}^{2}}\left[r_{h}^{2} f^{\prime \prime}\left(r_{h}\right)+4 r_{h} f^{\prime}\left(r_{h}\right)-2\right]\right) .
\end{gathered}
$$

Multiplying this equation by $d V=4 \pi r_{h}^{2} d r_{h}$, one gets

$$
\begin{aligned}
& P\left(r_{h}\right) d V=\frac{6 \gamma-1}{(4 \gamma-1)} \frac{f^{\prime}\left(r_{h}\right)}{4 \pi} d\left(\frac{A}{4}\right) \\
& -\frac{6 \gamma-1}{2(4 \gamma-1)} d r_{h}[1 \\
& \left.\quad+\gamma\left(r_{h}^{2} f^{\prime \prime}\left(r_{h}\right)+4 r_{h} f^{\prime}\left(r_{h}\right)-2\right)\right],
\end{aligned}
$$

where $A=4 \pi r_{h}^{2}$. For the second term of the RHS of this equation we reach

$$
\begin{aligned}
& \frac{6 \gamma-1}{2(4 \gamma-1)}\left[1+\gamma\left(r_{h}^{2} f^{\prime \prime}\left(r_{h}\right)+4 r_{h} f^{\prime}\left(r_{h}\right)-2\right)\right] d r_{h} \\
& \quad=\frac{6 \gamma-1}{2(4 \gamma-1)}\left[(1-2 \gamma) d r_{h}+\gamma d\left(r_{h}^{2} f^{\prime}\left(r_{h}\right)\right)\right. \\
& \left.\quad+2 r_{h} f^{\prime}\left(r_{h}\right) d r_{h}\right] .
\end{aligned}
$$

Now, bearing the $f\left(r_{h}\right)=0$ condition in mind, since $r_{h} f^{\prime}\left(r_{h}\right)=(d[r f(r)] / d r-f(r))_{r=r_{h}}$, one can simplify the RHS of the recent equation and take integral from that to get (11). Therefore, (17) can be written as follows:

$$
P\left(r_{h}\right) d V=\frac{6 \gamma-1}{4 \gamma-1} \frac{f^{\prime}\left(r_{h}\right)}{4 \pi} d\left(\frac{A}{4}\right)-d E .
$$


Since $T=f^{\prime}\left(r_{h}\right) / 4 \pi$ is the horizon temperature, comparing this equation with the first law of thermodynamics $(P d V=$ $T d S-d E)$ [7], one gets $d S=((6 \gamma-1) /(4 \gamma-1)) d(A / 4)$ which finally leads to

$$
S=\left(1+\frac{2 \gamma}{4 \gamma-1}\right) S_{0}
$$

where $S_{0}=A / 4$ is the Bekenstein entropy, for the horizon entropy. It is now obvious that, in the $\gamma \rightarrow 0$ limit, we have $\widetilde{S} \equiv$ $(2 \gamma /(4 \gamma-1)) S_{0} \rightarrow 0$, and thus the Einstein result (the Bekenstein entropy) is recovered. Indeed, as authors have shown in $[2,7-11]$, terms other than the Einstein tensor in modified gravities modify the Bekenstein limit of the system entropy in agreement with our result $(\widetilde{S})$. In addition, since entropy is a positive quantity, the Rastall dimensionless parameter should meet either $\gamma<1 / 6$ or $\gamma>1 / 4$. Here, the energy-momentum conservation law is not valid and therefore the energy and entropy terms differ from those of the Einstein theory [7]. In order to have a better view about our results, we compare our results with those of the Einstein theory [7]. Bearing the $\widetilde{S}$ and $\widetilde{E}$ definitions in mind, one can rewrite (19) as

$$
P\left(r_{h}\right) d V=T d S_{0}-d E_{0}+(T d \widetilde{S}-d \widetilde{E}) .
$$

$T d S_{0}-d E_{0} \equiv d W_{0}$ is the amount of work done during a hypothetical displacement $d r_{h}$ to the horizon in the Einstein framework [7]. Therefore, if we decompose the work term $\left(P\left(r_{h}\right) d V\right)$ as $P\left(r_{h}\right) d V=d \widetilde{W}+d W_{0}$, then we reach

$$
d \widetilde{W} \equiv P\left(r_{h}\right) d V-d W_{0}=T d \widetilde{S}-d \widetilde{E},
$$

which denotes the additional work done in the Rastall theory in comparison with the Einstein theory. Finally, it is also useful to mention here that, as a desired result, in the absence of the Rastall term $(\lambda=\gamma=0)$, we have $d \widetilde{S}=d \widetilde{E}=d \widetilde{W}=0$ meaning that the Einstein result is recovered [7].

\section{Concluding Remarks}

We saw that the Rastall theory of either $\gamma=1 / 6$ or $\gamma=$ $1 / 4$ is not allowed due to the fact that the Newtonian limit should be satisfied by the Rastall theory. Additionally, we used the unified first law of thermodynamics as well as the zerozero component of the Rastall field equations to generalize the Misner-Sharp mass of the static spherically symmetric spacetimes to the Rastall theory.

Moreover, bearing the obtained Misner-Sharp mass in mind, we started from the one-one component of the Rastall field equations and rewrote it as the first law of thermodynamics which helped us in finding an expression for the horizon entropy in this theory. Our study shows that a term other than the Einstein tensor in the gravitational field equations, the Rastall term $\left(\gamma g_{\mu \nu} R\right)$, modifies the Bekenstein limit. We also compared the obtained thermodynamic quantities, including entropy, energy, and work, with their counterparts in the Einstein case to have a better view about the obtained quantities. As we saw, in the $\gamma=\lambda=0$ limit, the results of the Einstein theory are obtainable.

\section{Competing Interests}

The authors declare no competing interests regarding the publication of this paper.

\section{Acknowledgments}

The work of H. Moradpour has been supported financially by Research Institute for Astronomy \& Astrophysics of Maragha (RIAAM) under research Project no. 1/4165-5. Ines G. Salako thanks African Institute for Mathematical Sciences (AIMS), 6 Melrose Road, Muizenberg 7945, South Africa, for partial support.

\section{References}

[1] T. Jacobson, "Thermodynamics of spacetime: the Einstein equation of state," Physical Review Letters, vol. 75, no. 7, pp. 1260 1263, 1995.

[2] C. Eling, R. Guedens, and T. Jacobson, "Nonequilibrium thermodynamics of spacetime," Physical Review Letters, vol. 96, no. 12, 121301, 4 pages, 2006.

[3] C. W. Misner and D. H. Sharp, "Relativistic equations for adiabatic, spherically symmetric gravitational collapse," Physical Review, vol. 136, no. 2, pp. B571-B576, 1964.

[4] H. Maeda, "Final fate of spherically symmetric gravitational collapse of a dust cloud in Einstein-Gauss-Bonnet gravity," Physical Review D, vol. 73, no. 10, Article ID 104004, 17 pages, 2006.

[5] H. Maeda and M. Nozawa, "Generalized Misner-Sharp quasilocal mass in Einstein-Gauss-Bonnet gravity," Physical Review D, vol. 77, no. 6, Article ID 064031, 13 pages, 2008.

[6] R.-G. Cai, L.-M. Cao, Y.-P. Hu, and N. Ohta, "Generalized Misner-Sharp energy in $f(R)$ gravity," Physical Review D, vol. 80, no. 10, Article ID 104016, 2009.

[7] A. Paranjape, S. Sarkar, and T. Padmanabhan, "Thermodynamic route to field equations in Lanczos-Lovelock gravity," Physical Review D, vol. 74, no. 10, Article ID 104015, 2006.

[8] T. Padmanabhan, “Thermodynamical aspects of gravity: new insights," Reports on Progress in Physics, vol. 73, no. 4, Article ID 046901, 2010.

[9] T. Padmanabhan, "Equipartition of energy in the horizon degrees of freedom and the emergence of gravity," Modern Physics Letters A, vol. 25, no. 14, pp. 1129-1136, 2010.

[10] T. Padmanabhan, "Surface density of spacetime degrees of freedom from equipartition law in theories of gravity," Physical Review D, vol. 81, no. 12, Article ID 124040, 2010.

[11] M. Akbar and R.-G. Cai, "Thermodynamic behavior of field equations for $\mathrm{f}(\mathrm{R})$ gravity," Physics Letters $B$, vol. 648, no. 2-3, pp. 243-248, 2007.

[12] M. Akbar and R.-G. Cai, "Friedmann equations of FRW universe in scalar-ensor gravity, $\mathrm{f}(\mathrm{R})$ gravity and first law of thermodynamics," Physics Letters B, vol. 635, no. 1, pp. 7-10, 2006.

[13] M. Akbar and R. G. Cai, "Thermodynamic behavior of the Friedmann equation at the apparent horizon of the FRW universe," Physical Review D, vol. 75, Article ID 084003, 2007.

[14] R.-G. Cai and L.-M. Cao, "Unified first law and the thermodynamics of the apparent horizon in the FRW universe," Physical Review D, vol. 75, no. 6, Article ID 064008, 11 pages, 2007. 
[15] R.-G. Cai and L.-M. Cao, “Thermodynamics of apparent horizon in brane world scenario," Nuclear Physics B, vol. 785, no. 1-2, pp. 135-148, 2007.

[16] A. Sheykhi, B. Wang, and R.-G. Cai, "Thermodynamical properties of apparent horizon in warped DGP braneworld," Nuclear Physics B, vol. 779, no. 1-2, pp. 1-12, 2007.

[17] A. Sheykhi, B. Wang, and R.-G. Cai, "Deep connection between thermodynamics and gravity in Gauss-Bonnet braneworlds," Physical Review D, vol. 76, no. 2, Article ID 023515, 5 pages, 2007.

[18] A. Sheykhi, "Thermodynamical interpretation of gravity in braneworld scenarios," Journal of Cosmology and Astroparticle Physics, vol. 2009, no. 5, p. 19, 2009.

[19] A. Sheykhi, "Thermodynamics of apparent horizon and modified Friedmann equations," The European Physical Journal C, vol. 69, no. 1-2, pp. 265-269, 2010.

[20] A. Sheykhi, "Thermodynamics of interacting holographic dark energy with the apparent horizon as an IR cutoff," Classical and Quantum Gravity, vol. 27, no. 2, Article ID 025007, 8 pages, 2010.

[21] R. G. Cai and N. Ohta, "Horizon thermodynamics and gravitational field equations in Hořava-Lifshitz gravity," Physical Review D, vol. 81, no. 8, Article ID 084061, 2010.

[22] A. Sheykhi, "Thermodynamics of the apparent horizon in infrared modified Horava-Lifshitz gravity," Physical Review D, vol. 87, no. 2, Article ID 024022, 7 pages, 2013.

[23] A. Sheykhi, M. H. Dehghani, and R. Dehghani, "Horizon thermodynamics and gravitational field equations in quasitopological gravity," General Relativity and Gravitation, vol. 46, article 1679, 2014.

[24] P. Rastall, "Generalization of the Einstein theory," Physical Review D, vol. 6, pp. 3357-3359, 1972.

[25] S. Nojiri and S. D. Odintsov, "Gravity assisted dark energy dominance and cosmic acceleration," Physics Letters B, vol. 599, no. 3-4, pp. 137-142, 2004.

[26] G. Allemandi, A. Borowiec, M. Francaviglia, and S. D. Odintsov, "Dark energy dominance and cosmic acceleration in first-order formalism," Physical Review D, vol. 72, no. 6, Article ID 063505, 2005.

[27] T. Koivisto, "A note on covariant conservation of energymomentum in modified gravities," Classical and Quantum Gravity, vol. 23, no. 12, pp. 4289-4296, 2006.

[28] O. Bertolami, C. G. Böhmer, T. Harko, and F. S. Lobo, "Extra force in $f(R)$ modified theories of gravity," Physical Review D, vol. 75, no. 10, Article ID 104016, 5 pages, 2007.

[29] T. Harko and F. S. N. Lobo, "Generalized curvature-matter couplings in modified gravity," Galaxies, vol. 2, no. 3, pp. 410465, 2014.

[30] L. L. Smalley, "Variational principle for a prototype Rastall theory of gravitation," Il Nuovo Cimento B, vol. 80, no. 1, pp. 4248, 1984.

[31] C. E. M. Batista, M. H. Daouda, J. C. Fabris, O. F. Piattella, and D. C. Rodrigues, "Rastall cosmology and the $\Lambda \mathrm{cDM}$ model," Physical Review D, vol. 85, no. 8, Article ID 084008, 2012.

[32] G. W. Gibbons and S. W. Hawking, "Cosmological event horizons, thermodynamics, and particle creation," Physical Review D, vol. 15, no. 10, pp. 2738-2751, 1977.

[33] L. Parker, "Quantized fields and particle creation in expanding universes. II," Physical Review D, vol. 3, no. 2, p. 346, 1971, Physical Review D, vol. 3, no. 10, pp. 2546, 1971.

[34] L. H. Ford, "Gravitational particle creation and inflation," Physical Review D, vol. 35, no. 10, pp. 2955-2960, 1987.
[35] N. D. Birrell and P. C. W. Davies, Quantum Fields in Curved Space, Cambridge University Press, Cambridge, UK, 1982.

[36] H. Moradpour, "Thermodynamics of flat FLRW universe in Rastall theory," Physics Letters B, vol. 757, pp. 187-191, 2016.

[37] A. M. Oliveira, H. E. S. Velten, J. C. Fabris, and L. Casarini, "Neutron stars in Rastall gravity," Physical Review D, vol. 92, no. 4, Article ID 044020, 6 pages, 2015.

[38] C. E. M. Batista, J. C. Fabris, O. F. Piattella, and A. M. VelasquezToribio, "Observational constraints on Rastall's cosmology," European Physical Journal C, vol. 73, no. 5, pp. 1-7, 2013.

[39] J. C. Fabris, O. F. Piattella, D. C. Rodrigues, and M. H. Daouda, "Rastall's cosmology and its observational constraints," AIP Conference Proceedings, vol. 1647, no. 1, pp. 50-53, 2015.

[40] T. R. P. Caramês, M. H. Daouda, J. C. Fabris, A. M. Oliveira, O. F. Piattella, and V. Strokov, "The Brans-Dicke-Rastall theory," European Physical Journal C, vol. 74, article 3145, 2014.

[41] T. Caramês, M. H. Daouda, J. C. Fabris, A. M. Oliveira, O. F. Piattella, and V. Strokov, "A Rastall scalar-tensor theory," http:// arxiv.org/abs/1503.04882v1.

[42] J. C. Fabris, O. F. Piattella, D. C. Rodrigues, C. E. M. Batista, and M. H. Daouda, "Rastall cosmology," International Journal of Modern Physics: Conference Series, vol. 18, pp. 67-76, 2012.

[43] J. P. Campos, J. C. Fabris, R. Perez, O. F. Piattella, and H. Velten, "Does Chaplygin gas have salvation?" The European Physical Journal C, vol. 73, article 2357, 2013.

[44] J. C. Fabris, M. Hamani Daouda, and O. F. Piattella, "Note on the evolution of the gravitational potential in Rastall scalar field theories," Physics Letters B: Nuclear, Elementary Particle and High-Energy Physics, vol. 711, no. 3-4, pp. 232-237, 2012.

[45] J. C. Fabris, "Conservation laws in gravitation and cosmology," https://arxiv.org/abs/1208.4649vl.

[46] I. G. Salako and A. Jawad, "Bianchi type-III models with anisotropic dark energy in Brans-Dicke-Rastall theory," Astrophysics and Space Science, vol. 359, article 46, 2015.

[47] G. F. Silva, O. F. Piattella, J. C. Fabris, L. Casarini, and T. O. Barbosa, "Bouncing solutions in Rastall's theory with a barotropic fluid," Gravitation and Cosmology, vol. 19, no. 3, pp. 156-162, 2013.

[48] M. Capone, V. F. Cardone, and M. L. Ruggiero, "The possibility of an accelerating cosmology in Rastall's theory," Journal of Physics: Conference Series, vol. 222, no. 1, Article ID 012012, 2010.

[49] Ph. Brax, C. van de Bruck, and A. C. Davis, "A chameleon primer," http://arxiv.org/abs/0706.1024.

[50] E. R. Bezerra de Mello, J. C. Fabris, and B. Hartmann, "AbelianHiggs strings in Rastall gravity," Classical and Quantum Gravity, vol. 32, no. 8, Article ID 085009, 2015.

[51] H. Moradpour and N. Sadeghnezhad, "Traversable asymptotically flat wormholes in Rastall gravity," https://arxiv.org/abs/ 1606.00846 . 

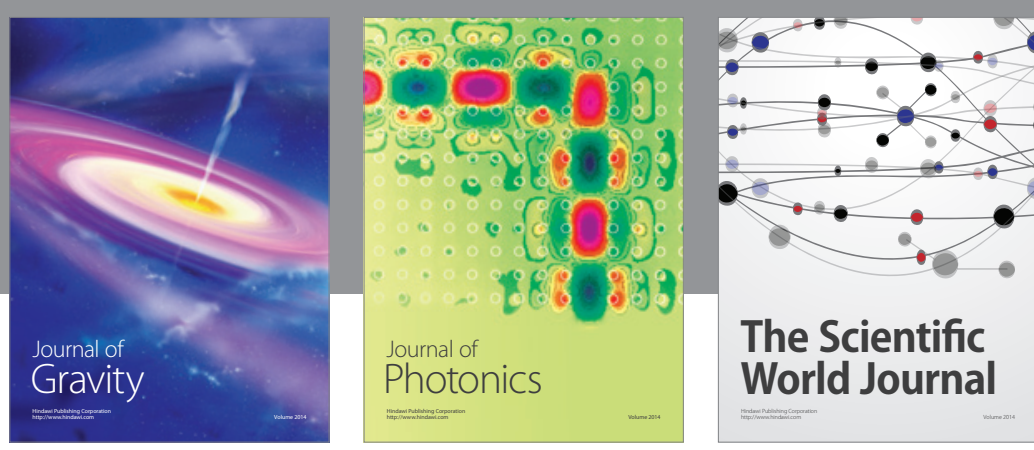

The Scientific World Journal
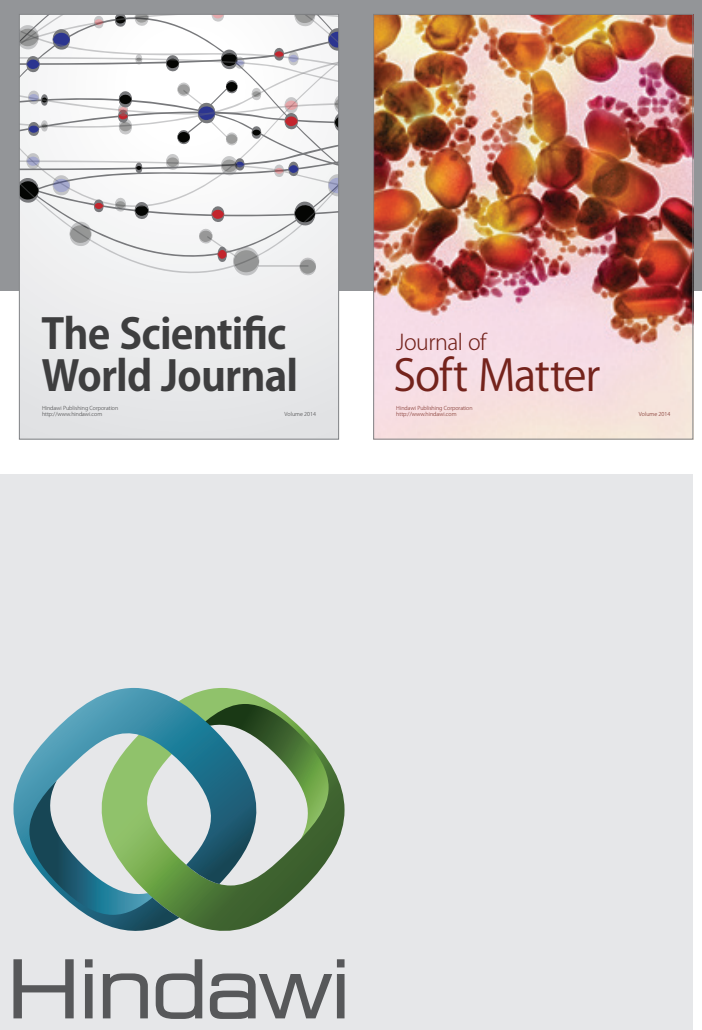

Submit your manuscripts at

http://www.hindawi.com

nternational Journal of

Statistical Mechanics
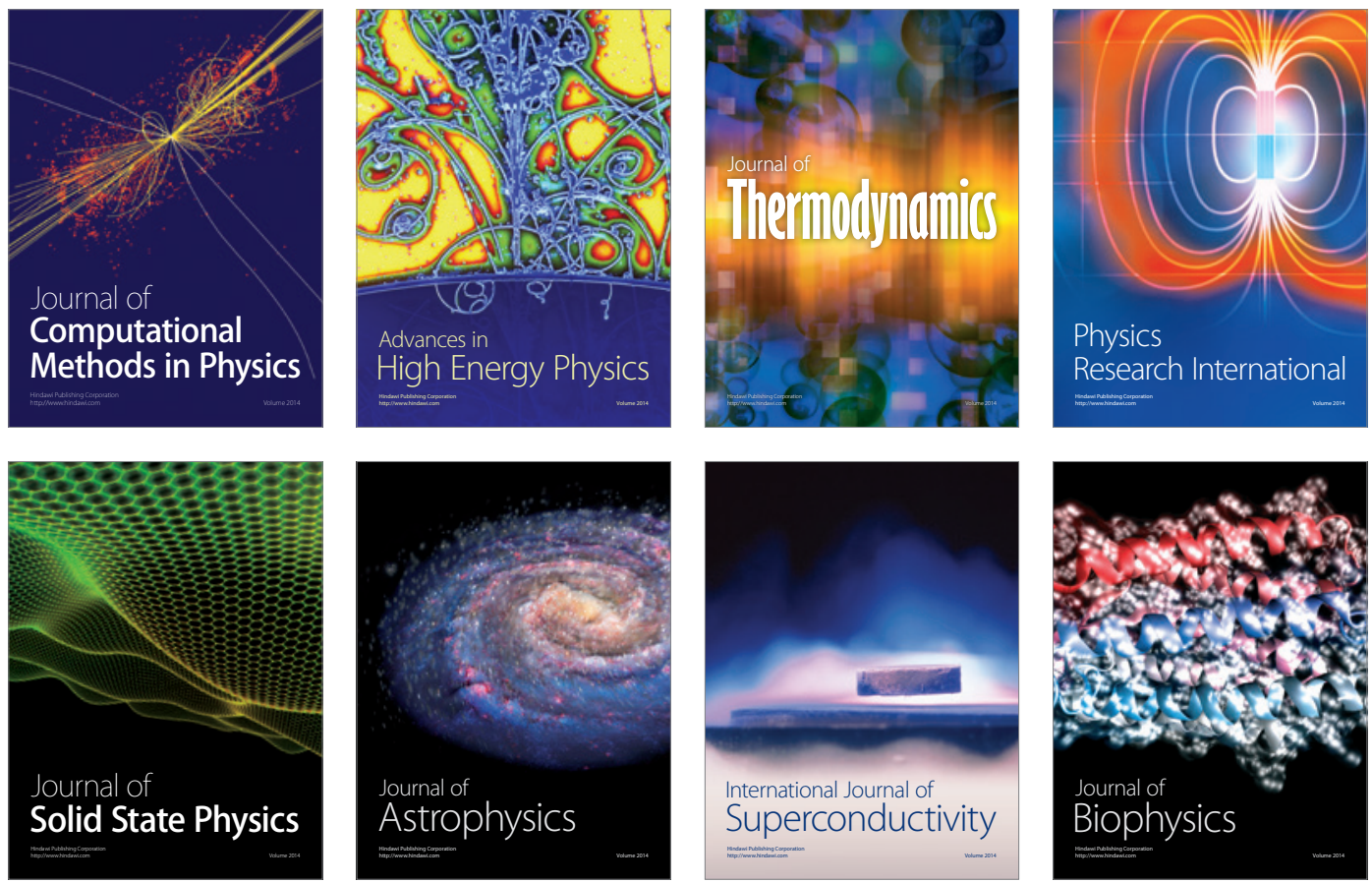
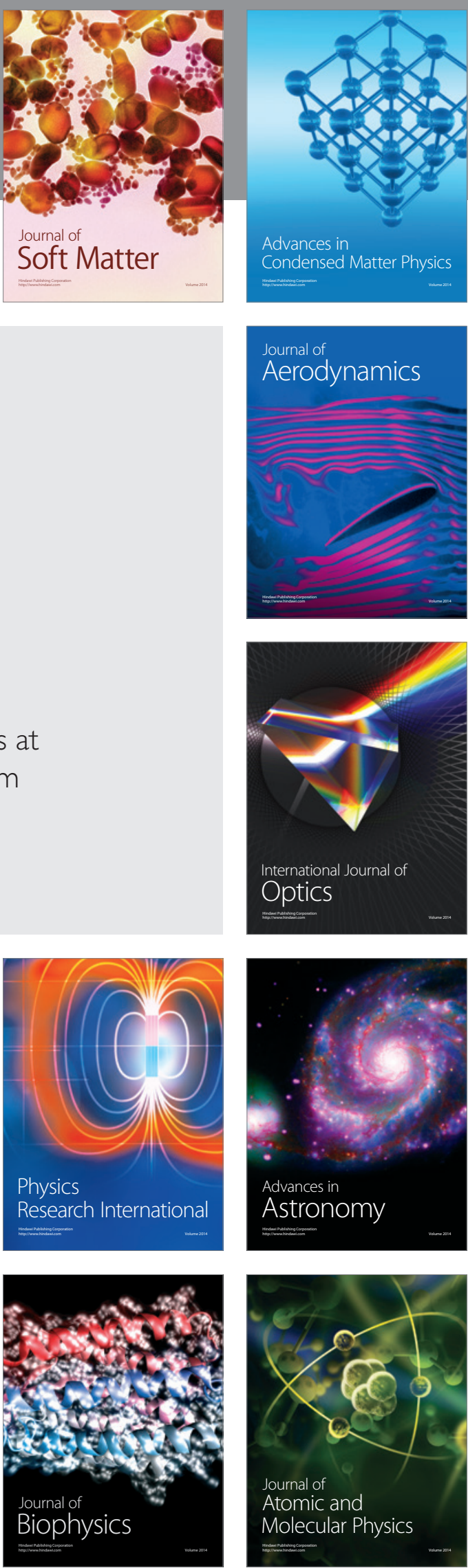